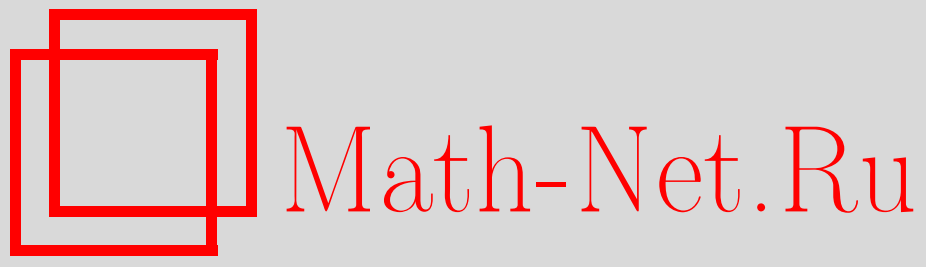

И. Т. Хабибуллин, Е. В. Гудкова, Краевые условия для многомерных интегрируемых уравнений, Функи. анализ и его прил., 2004, том 38, выпуск $2,71-83$

DOI: https://doi.org/10.4213/faa109

Использование Общероссийского математического портала MathNet.Ru подразумевает, что вы прочитали и согласны с пользовательским соглашением

http://www. mathnet.ru/rus/agreement

Параметры загрузки:

IP: 18.208 .226 .222

26 апреля 2023 г., 18:26:24

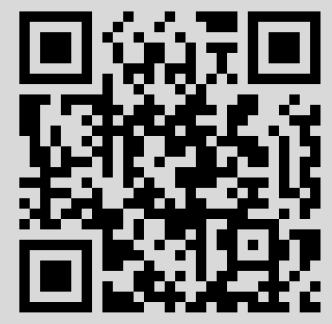


Функииональный анализ и его приложения

2004, т. 38, вып. 2, с. 71-83

УДК 517.9

\title{
Краевые условия для многомерных интегрируемых уравнений*
}

\author{
(c) 2004. И. Т. ХАБиБУллин, Е. В. ГудковА
}

Работа Е. К. Склянина [1] вызвала всплеск интереса к краевым условиям для нелинейных интегрируемых уравнений, согласованным со свойством интегрируемости. За последние полтора десятилетия для моделей размерности $1+1$, т. е. для уравнений с одной пространственной и одной временно́й переменной были найдены эффективные алгоритмы поиска таких краевых условий, использующие законы сохранения и гамильтонову структуру [1], высшие симметрии уравнения [2], а также непосредственно пару Лакса [3-5]. Хотя среди всех допустимых краевых условий интегрируемые составляют достаточно узкий класс, они могут служить основой для оценки влияния произвольных краевых условий, например, методами теории возмущений. Отметим, что для уравнений с двумя и более пространственными переменными проблема краевых условий, согласующихся со свойством интегрируемости, остается мало исследованной. В качестве примеров можно упомянуть лишь несколько типов краевых условий для уравнения Ишимори, найденных в [6], и так называемые обобщенные цепочки Тоды - редукции бесконечной двумеризованной цепочки Тоды, связанные с классическими сериями алгебр Ли конечного роста (см., например, монографию [7]). Они получаются из бесконечной цепочки при наложении условий обрыва (краевых условий по дискретной переменной), согласованных с интегрируемостью. Любопытное наблюдение, стимулировавшее написание настоящей работы, состоит в том, что если на бесконечную цепочку Тоды

$$
u_{x t}(n)=\exp \{u(n-1)-u(n)\}-\exp \{u(n)-u(n+1)\},
$$

налагается интегрируемое условие обрыва вида $F(u(0), u(-1))=0$, то второе уравнение пары Лакса этой цепочки

$$
\begin{gathered}
\phi(n+1)=\left(D_{x}+u_{x}(n)\right) \phi(n), \\
\phi_{x t}(n)=-u_{x}(n) \phi_{t}(n)-\exp \{u(n-1)-u(n)\} \phi(n)
\end{gathered}
$$

приобретает дополнительную инволюцию в точке $n=0$. Здесь $D_{x}$ - оператор дифференцирования по $x$. Прежде чем формулировать точное утверждение, заметим, что уравнение (1) инвариантно относительно преобразования $x \leftrightarrow t$; поэтому наряду с системой (2), (3) пару Лакса для цепочки (1) доставляет также следующая пара уравнений:

$$
\begin{gathered}
\psi(n+1)=\left(D_{t}+u_{t}(n)\right) \psi(n), \\
\psi_{x t}(n)=-u_{t}(n) \psi_{x}(n)-\exp \{u(n-1)-u(n)\} \psi(n),
\end{gathered}
$$

* Работа поддержана грантами РФФИ (04-01-00190), Минобразования (Е02-1.0-77) и Программы поддержки ведущих научных школ (НШ-1716.2003.1). 
причем эти две пары не связаны одна с другой преобразованием подобия. Однако они становятся подобными при наложении дополнительных условий связи между коэффициентами операторов в некоторой фиксированной точке. Точнее, верно следующее утверждение:

ПрЕДЛОЖЕНИЕ 1. Пусть существует дифберенциальный оператор вида $M=a D_{x}^{2}+b D_{x}+c$, такой, что при $n=0$ для любого решения $\psi$ уравнения (5) функиия $\phi=M \psi$ является решением уравнения (3). Тогда переменные $и(0)$, u(-1) удовлетворяют одному из следующих соотношений:

$$
\begin{aligned}
e^{u(-1)} & =0, \\
u(-1) & =0 \\
u(-1) & =-u(0), \\
u_{x}(-1) & =-u_{t}(0) e^{-u(0)-u(-1)} .
\end{aligned}
$$

При этом соответствующий оператор $M$ имеет вид

$$
\begin{aligned}
& M_{1}=a_{0} e^{u} D_{x}^{2}+\left(b_{0} e^{u}+a_{0} u_{x} e^{u}\right) D_{x}, \\
& M_{2}=a_{0}\left(e^{u} D_{x}^{2}+u_{x} e^{u} D_{x}\right), \\
& M_{3}=a_{0} e^{u} D_{x} \\
& M_{4}=a_{0}\left(e^{u} D_{x}^{2}+u_{x} e^{u} D_{x}+e^{-u}\right) .
\end{aligned}
$$

где $a_{0}, b_{0}$ - произвольные числовые параметры, $u=u(0)$.

Отметим, что оператор $M_{1}$ является линейной комбинацией операторов $M_{2}$ и $M_{3}$.

СХЕМА ДОКАЗАТЕЛЬСТВА ПРЕДЛОЖЕНИЯ 1. Дифференцируя равенство $\phi=$ $M \psi$ по $x$ и $t$, получаем

$$
\phi_{x t}=M_{x t} \psi+M_{x} \psi_{t}+M_{t} \psi_{x}+M \psi_{x t} .
$$

Выразим в этом равенстве переменные $\phi, \phi_{x}, \phi_{t}, \phi_{x t}, \ldots$ в силу уравнения (3) и соотношения $\phi=M \psi$ через динамические переменные $\psi, \psi_{x}, \psi_{t}, \psi_{x x}, \psi_{t t}, \ldots$. Приравнивая теперь в равенстве (8) коэффициенты при этих динамических переменных, получаем переопределенную систему уравнений на коэффициенты $a, b, c$ искомого оператора $M$. Условия разрешимости полученной системы имеют вид дифференциальных связей между переменными $u(-1)$ и $u(0)$, которые и приведены выше в предложении 1.

Нетрудно видеть, что равенства (6) есть не что иное, как хорошо известные интегрируемые условия обрыва цепочки Тоды (в этом списке отсутствует условие обрыва, соответствующее серии $D_{n}$, поскольку оно связано с оператором $M$ более высокого порядка). Поэтому предложение 1 можно положить в основу предварительного теста для проверки краевого условия на интегрируемость. А именно, мы будем считать, что краевое условие проходит тест на совместимость с L-A-парой, если соответствующие операторы двух неподобных пар Лакса становятся подобными при наложении этого краевого условия. Для 1+1-мерных интегрируемых уравнений аналогичный тест имеет простое объяснение: наличие дополнительной дискретной симметрии в граничной точке позволяет решить уравнение Дегаспериса-Манакова-Сантини [8], описывающее временну́ю динамику матрицы рассеяния, сведением к матричной задаче Римана. Для $1+2$-мерных уравнений наш подход позволяет просто и эффективно 
классифицировать интегрируемые краевые условия, пользуясь только L-A-парой уравнения. Ниже мы используем предлагаемый операторный тест для отыскания краевых условий для нескольких популярных многомерных уравнений. В частности, в $\S 1,5,6$ приведены новые типы краевых условий для уравнения Кадомцева-Петвиашвили (KП) и его модификации (мКП) и уравнения Веселова-Новикова. Показано, что любое из трех краевых условий $\left.w\right|_{y=0}=0$ и $\left.\left(v_{x} \pm \alpha w\right)\right|_{y=0}=0$ совместимо с уравнением КП $v_{\tau}+v_{x x x}-6 v v_{x}=-3 \alpha^{2} w_{y}$, $w_{x}=v_{y}$, где $\alpha$ принимает одно из двух значений $\alpha=i, \alpha=1$. В 22 проверено, что эти краевые условия совместимы со всеми однородными высшими симметриями нечетного порядка уравнения КП (см. предложение 3). В третьем и четвертом параграфах кратко обсуждается связь краевых условий с высшими симметриями и преобразованием Беклунда.

\section{§1. Краевые условия для уравнения КП}

В этом параграфе в качестве основной модели мы рассматриваем уравнение КП1 (все формулы ниже легко пересчитываются на случай уравнения КП2, которое связано с КП1 заменой $y \rightarrow i y, w \rightarrow-i w)$

$$
\begin{aligned}
v_{\tau}+v_{x x x}-6 v v_{x} & =3 w_{y}, \\
w_{x} & =v_{y},
\end{aligned}
$$

имеющее приложения в ряде физических задач, например, в физике плазмы и теории океанских волн [9]. Пара Лакса для этого уравнения, как известно из [10-12], имеет вид

$$
\begin{aligned}
\phi_{x x} & =i \phi_{y}+v \phi \\
\phi_{\tau} & =-4 \phi_{x x x}+6 v \phi_{x}+3\left(v_{x}+i w\right) \phi .
\end{aligned}
$$

Уравнение (9) не изменяет своего вида при одновременной смене знака у переменных $y$ и $w$; поэтому система уравнений

$$
\begin{aligned}
\psi_{x x} & =-i \psi_{y}+v \psi, \\
\psi_{\tau} & =-4 \psi_{x x x}+6 v \psi_{x}+3\left(v_{x}-i w\right) \psi
\end{aligned}
$$

определяет пару Лакса для уравнения КП, сопряженную к паре (10), (11). Нетрудно проверить, что, вообще говоря, уравнения (11) и (13) не переводятся одно в другое преобразованием подобия, т. е. не существует подстановки вида $\phi=M \psi$ или вида $\psi=M \phi$, определяемой дифференциальным оператором $M$, которая связывала бы эти два уравнения. В то же время если на коэффициенты уравнений наложить дополнительные условия связи, то уравнения становятся подобными. Имеет место следующее утверждение:

ПРЕДЛОЖЕНИЕ 2. Пусть существует дифберенииалъный оператор вида $M=a D_{x}^{2}+b D_{x}+c$, такой, что при $y=0$ для любого решения $\psi$ уравнения (13) функиия $\phi=M \psi$ является решением уравнения (11). Тогда выполняется одно из следующих соотношений:

$$
\begin{gathered}
\left.w\right|_{y=0}=0, \\
\left.\left(v_{x}-i w\right)\right|_{y=0}=0, \\
\left.\left(w_{\tau}-2 v_{x x y}+6 i v_{y y x}+6 v_{x} w-6 v w_{x}-6 i w^{2}-12 c v_{y}\right)\right|_{y=0}=0,
\end{gathered}
$$


где функиия $c=c(x, \tau)$ определяется из уравнения $c_{x}=\left.\left(-v_{x}+\frac{i}{2} w\right)\right|_{y=0}$. При этом соответствующий оператор $M$ имеет вид

$$
\begin{aligned}
& M=1, \\
& M=D_{x}, \\
& M=D_{x}^{2}+c .
\end{aligned}
$$

ДокАЗАтЕЛьство. Полагая $A_{1}=-4 D_{x}^{3}+6 v D_{x}+3\left(v_{x}+i w\right), A_{2}=-4 D_{x}^{3}+$ $6 v D_{x}+3\left(v_{x}-i w\right)$, перепишем уравнения (11) и (13) в виде

$$
\phi_{\tau}=A_{1} \phi, \quad \psi_{\tau}=A_{2} \psi \text {. }
$$

Продифференцируем соотношение $\phi=M \psi$ по $\tau$ и, воспользовавшись уравнениями $(16)$, запишем результат в виде $A_{1} M \psi=\left(M_{\tau}+M A_{2}\right) \psi$. В силу произвольности $\psi$ отсюда имеем следующее операторное соотношение:

$$
M_{\tau}=A_{1} M-M A_{2} .
$$

Сравнивая в этом равенстве коэффициенты при различных степенях оператора $D_{x}$, получаем сильно переопределенную систему уравнений для отыскания неизвестных функций $a, b, c$ :

$$
\begin{aligned}
& D_{x}^{4}: a_{x}=0, \\
& D_{x}^{3}: b_{x}=0, \\
& D_{x}^{2}: a_{\tau}+12 c_{x}+6 a\left(2 v_{x}-i w\right)=0, \\
& D_{x}: b_{\tau}+12 c_{x x}+6 a\left(2 v_{x x}-i w_{x}\right)+6 b\left(v_{x}-i w\right)=0, \\
& D_{x}^{0}: c_{\tau}+4 c_{x x x}-6 v c_{x}+3 a\left(v_{x x x}-i w_{x x}\right)+3 b\left(v_{x x}-i w_{x}\right)-6 i c w=0 .
\end{aligned}
$$

Продифференцируем по $x$ третье из уравнений (18) и вычтем результат из четвертого уравнения. Получим, что $b_{\tau}+6 b\left(v_{x}-i w\right)=0$. Отсюда в силу того, что $b(\tau)=b(0) \exp \left(-6 \int_{0}^{\tau}\left(v_{x}-i w\right) d \tau^{\prime}\right)$, имеем либо (i) $b \equiv 0$, либо (ii) $b(\tau) \neq 0$ для любого $\tau$. Кроме того, выполняется равенство

$$
c_{x x}=-a\left(v_{x x}-\frac{i}{2} w_{x}\right) .
$$

Предположим, что имеет место случай (i). Тогда пятое уравнение системы (18) принимает вид

$$
c_{\tau}+4 c_{x x x}-6 v c_{x}+3 a\left(v_{x x x}-i w_{x x}\right)-6 i c w=0 .
$$

Функция $c=c(x, \tau)$ удовлетворяет одновременно двум уравнениям (19) и (20). При проверке совместности этих уравнений приходим к развилке (j) $a=0$ и $(\mathrm{jj}) a \neq 0$. В первом случае имеем $c_{x}=0$ и $c_{\tau}=6 i w c$ и потому $w_{x}=0$. Здесь мы приходим к краевому условию $\left.w\right|_{y=0}=w_{0}(\tau)$, где $w_{0}(\tau)-$ произвольная функция, но этот произвол убирается при помощи преобразования $w \rightarrow w-$ $w_{0}(\tau)$, не изменяющего уравнение КП. Тем самым краевое условие приводится к виду (14.1). Во втором случае, когда $a \neq 0$, можно положить $a=1$, и тогда граничное условие принимает вид

$$
w_{\tau}=2 v_{x x y}-6 i v_{y y x}-6 v_{x} w+6 v w_{x}+6 i w^{2}+12 c v_{y},
$$

где $c$ определяется из уравнения $c_{x}=-v_{x}+\frac{i}{2} w$, т. е. совпадает с (14.3). 
В случае (ii) имеем $6\left(v_{x x}-i w_{x}\right)=-(\ln b)_{x \tau}=0$. Теперь третье и четвертое уравнения системы (18) примут вид $a_{\tau}=-12 c_{x}-6 a v_{x}$ и $b_{\tau}=-12 c_{x x}-6 a v_{x x}$. Отсюда следует, что $b_{\tau}=a_{x \tau}=0$, но тогда из условия $b_{\tau}+6 b\left(v_{x}-i w\right)=0$ получаем равенство $v_{x}-i w=0$, совпадающее с (14.2). При этом уравнения (19) и $(20)$ совместны, лишь когда $a=0$. Отсюда имеем $c_{x}=0$ и $c_{\tau}=6 i w c$, где вообще говоря, $w_{x} \neq 0$. Поэтому $c=0$ и оператор $M$ принимает вид $(15.2)$.

Поменяв местами уравнения (11) и (13) (т. е. функции $\psi$ и $\phi$ ), находим еще два краевых условия

$$
\begin{gathered}
\left.\left(v_{x}+i w\right)\right|_{y=0}=0 \\
\left.\left(w_{\tau}-2 v_{x x y}-6 i v_{y y x}-6 v_{x} w+6 v w_{x}-6 i w^{2}-12 c v_{y}\right)\right|_{y=0}=0
\end{gathered}
$$

где $c_{x}=v_{x}+\frac{i}{2} w$.

Предложение 2 показывает, что краевые условия (14.1)-(14.5), налагаемые на уравнение КП, проходят предварительный операторный тест на совместимость с интегрируемостью. При этом, если краевые условия (14.1), (14.2) и (14.4) формулируются просто, то два других трудно обозримы. Возможно, они упрощаются при дальнейших редукциях. Ниже мы сосредоточимся на условиях (14.1), (14.2) и (14.4). Покажем, что они согласуются с такими ингредиентами интегрируемости, как высшие симметрии и преобразование Беклунда. Метод обратной задачи рассеяния (см., например, [13]), по-видимому, переносится и на начально-краевые задачи с краевыми условиями вида (14.1)-(14.5), однако в данной работе проблема построения решений краевой задачи не рассматривается.

\section{2. Мастер-симметрия и краевые условия}

Для компактного описания иерархии высших симметрий уравнения удобно пользоваться понятием мастер-симметрии. Мастер-симметрия уравнения $u_{\tau}=$ $f\left(u, u_{x}, u_{x x}, \ldots\right)$ определяется как уравнение вида $u_{t}=g\left(u, u_{x}, u_{x x}, \ldots\right)$, такое, что для любой симметрии $u_{\tau_{1}}=f_{1}\left(u, u_{x}, u_{x x}, \ldots\right)$ этого уравнения уравнение $u_{\tau_{2}}=f_{2}\left(u, u_{x}, u_{x x}, \ldots\right)$, где $f_{2}=\frac{\partial}{\partial t} f_{1}-\frac{\partial}{\partial \tau_{1}} g$, также является симметрией. В частности, уравнение (см., например, [14])

$$
\begin{aligned}
& v_{t_{1}}=\frac{1}{2} y v_{y}-2 y v v_{y}-y w v_{x}-\frac{1}{2} y D_{x}^{-2} v_{y y y}+\frac{1}{8} x v_{3} \\
& \quad-\frac{3}{4} x v v_{x}-\frac{3}{8} x D_{x}^{-1} v_{y y}+\frac{1}{4} i v_{y}-\frac{3}{4} D_{x}^{-2} v_{y y}-\frac{1}{4} v_{x} D_{x}^{-1} v-v^{2}
\end{aligned}
$$

является мастер-симметрией уравнения КП. Эволюция собственных функций $\phi$ и $\psi$ уравнений $(10)$ и (12) по $t_{1}$ определяется в силу условий

$$
\phi_{t_{1}}=B_{1} \phi, \quad \psi_{t_{1}}=B_{2} \psi
$$

где $B_{1}=-i y D_{x}^{4}+\frac{1}{2} x D_{x}^{3}+(1+2 i y v) D_{x}^{2}+\left(2 i y v_{1}-\frac{3}{4} x v-\frac{1}{4} D_{x}^{-1} v-y D_{x}^{-1} v_{y}\right) D_{x}+$ $i y v_{2}-\frac{3}{8} x v_{1}-\frac{3}{4} v-\frac{1}{2} y v_{y}-\frac{3}{8} i x D_{x}^{-1} v_{y}-\frac{1}{4} i D_{x}^{-2} v_{y}-\frac{1}{2} i y D_{x}^{-2} v_{y y}-i y v^{2}$ и $B_{2}=$ $i y D_{x}^{4}+\frac{x}{2} D_{x}^{3}+\left(\frac{1}{2}-2 i y v\right) D_{x}^{2}+\left(-2 i y v_{1}-\frac{3}{4} x v-\frac{1}{4} D_{x}^{-1} v-y D_{x}^{-1} v_{y}\right) D_{x}-i y v_{2}-\frac{3}{8} x v_{1}-$ $\frac{1}{4} v-\frac{1}{2} y v_{y}+\frac{3}{8} i x D_{x}^{-1} v_{y}+\frac{1}{4} i D_{x}^{-2} v_{y}+\frac{1}{2} i y D_{x}^{-2} v_{y y}+i y v^{2}$.

Введем зависимость от высших времен $\tau_{n}$ по правилу

$$
\phi_{\tau_{n}}=A_{1}^{(n)} \phi, \quad \psi_{\tau_{n}}=A_{2}^{(n)} \psi,
$$


где $A_{1}^{(0)}=A_{1}$ и $A_{2}^{(0)}=A_{2}$, а при $n>0$ операторы $A_{j}^{(n)}, j=1,2$, определяются рекуррентным способом:

$$
A_{j}^{(n+1)}=\frac{\partial}{\partial t_{1}} A_{j}^{(n)}-\frac{\partial}{\partial \tau_{n}} B_{j}+\left[A_{j}^{(n)}, B_{j}\right] .
$$

Нетрудно проверить, что условие совместности первого из уравнений (23) и уравнения (10), а также условие совместности второго из уравнений (23) и уравнения (12) определяют одну и ту же высшую симметрию нечетного порядка $2 n+3$. Покажем, что эта симметрия совместима с краевыми условиями (14.1), (14.2), (14.4).

ПРЕДЛОЖЕНИЕ 3. Пусть выполнено одно из следующих краевых условий

$$
\begin{aligned}
\left.w\right|_{y=0} & =0, \\
v_{x}-\left.i w\right|_{y=0} & =0, \\
v_{x}+\left.i w\right|_{y=0} & =0 .
\end{aligned}
$$

Тогда система уравнений (23) при $y=0$ и при всех $n \geqslant 0$ допускает coответствующее условие редукиии

$$
\begin{aligned}
\phi & =\psi, \\
\phi & =D_{x} \psi, \\
\psi & =D_{x} \phi .
\end{aligned}
$$

Другими словами, условие редукции $\phi=M \psi$ (либо $\psi=M \phi)$, наложенное при $y=0$, совместимо с системой уравнений (23) при всех $n \geqslant 0$, если выполняется соответствующее краевое условие (см. предложение 2). Это означает, что краевые условия совместимы со всеми однородными высшими симметриями нечетного порядка.

ДОКАЗАТЕЛЬСТВО ПРЕДЛОЖЕНИЯ 3. Для определенности рассмотрим случай краевого условия (25.2) (остальные случаи доказываются аналогично). Тогда $M=D_{x}$. При этом, как легко заметить, равенство $\phi=D_{x} \psi$ согласуется с системой (22) при $y=0$ и $v_{x}=i w$. Действительно, $\left.B_{1}\right|_{y=0}=\frac{1}{2} x D_{x}^{3}+D_{x}^{2}+D_{x} h$ и $\left.B_{2}\right|_{y=0}=\frac{1}{2} x D_{x}^{3}+\frac{1}{2} D_{x}^{2}+h D_{x}$, где $h=-\frac{1}{4}\left(3 x v+D^{-1} v\right)$. Отсюда легко получить, что при $y=0$ выполняется равенство $B_{1} D_{x}=D_{x} B_{2}$.

Докажем теперь предложение 3 индукцией по $n$. Справедливость утверждения при $n=0$ следует из предложения 2 . Пусть оно верно при $n$, т. е. $A_{1}^{(n)} D_{x}=$ $D_{x} A_{2}^{(n)}$. Из рекуррентной формулы (24) вытекает, что

$$
\begin{aligned}
A_{1}^{(n+1)} D_{x}-D_{x} A_{2}^{(n+1)}=\frac{\partial}{\partial t} & \left(A_{1}^{(n)} D_{x}-D_{x} A_{2}^{(n)}\right)-\frac{\partial}{\partial \tau_{n}}\left(B_{1} D_{x}-D_{x} B_{2}\right) \\
& +\left[A_{1}^{(n)}, B_{1}\right] D_{x}-D_{x}\left[A_{2}^{(n)}, B_{2}\right] .
\end{aligned}
$$

Полагая в полученном равенстве $y=0$ и $v_{x}=i w$ и пользуясь тем, что $A_{1}^{(n)} D_{x}=$ $D_{x} A_{2}^{(n)}$ и $B_{1} D_{x}=D_{x} B_{2}$, приходим к равенству $A_{1}^{(n+1)} D_{x}=D_{x} A_{2}^{(n+1)}$. Предложение 3 доказано. 


\section{§3. Обрывы цепочки Тоды и уравнение КП}

Рассмотрим симметрию уравнения КП, заданную в виде условия совместности линейных уравнений (3) и (11). Для этих уравнений переменная $n$ служит параметром; поэтому можно считать, что $n=0$. В терминах новых переменных $r=e^{-u(0)}$ и $s=e^{u(-1)}$, вводимых для сокращения формул, искомая симметрия имеет следующий вид:

$$
v_{t}=-2(r s)_{x}, \quad i w_{t}=2\left(s_{x x} r-s r_{x x}\right) .
$$

Здесь нелокальные переменные $r, s$ определяются из условий

$$
\begin{aligned}
& r_{\tau}=-4 r_{x x x}+6 v r_{x}+3\left(v_{x}+i w\right) r, \\
& s_{\tau}=-4 s_{x x x}+6 v s_{x}+3\left(v_{x}-i w\right) s .
\end{aligned}
$$

Отметим, что последние два уравнения совпадают с уравнениями (11) и (13). Кроме того, здесь прослеживается некая двойственность между уравнением КП и цепочкой Тоды. Дело в том, что эта же самая система соотношений (27)-(29) задает и симметрию цепочки Тоды. При этом собственно симметрией будет пара уравнений (28), (29), а соотношения (27) уже рассматриваются как способ введения нелокальностей $v, w$ (см. [15]). Упомянутая двойственность удивительным образом проявляет себя на уровне краевых условий. А именно, интегрируемое краевое условие для уравнения КП совместимо с симметрией (27) только в том случае, когда на цепочку (1) наложено в точке $n=0$ соответствующее (двойственное) условие обрыва. Обсудим этот факт более подробно на примере краевого условия (14.2), для которого двойственным является условие обрыва вида $u(-1)=0$ цепочки Тоды. Здесь фактически утверждается, что фазовые потоки, задаваемые уравнениями (9) и (27), коммутируют на многообразии $S$, определяемом равенством $\left.\left(v_{x}-i w\right)\right|_{y=0}=0$ и его дифференциальными следствиями по $x$ и по $\tau$, только при наложении дополнительного условия $s=e^{u(-1)}=$ const, что с точностью до сдвигов переменной $u(n)$, которые, очевидно, не изменяют саму цепочку, равносильно условию $s=1$ или условию $u(-1)=0$. Действительно, дифференцируя краевое условие $\left.\left(v_{x}-i w\right)\right|_{y=0}=0$ по $t$, в силу симметрии $(27)$ получим равенство

$$
\left.\frac{d}{d t}\left(v_{x}-i w\right)\right|_{y=0}=-4\left(r s_{x}\right)_{x}
$$

показывающее, что для коммутирования потоков на $S$ необходимо выполнение условия $\left(r s_{x}\right)_{x}=0$. Это последнее соотношение согласуется с динамикой по $\tau$ лишь при $r s_{x}=0$. Если далее предположить, что $r=0$, то из $(27)$ мгновенно получаем лишние условия связи типа $v_{t}=0, w_{t}=0$ и т. Д. Поэтому полагаем $s=1$. Дифференцирование этого равенства по $t$ в силу (27) не приводит к появлению новых связей; иначе говоря, при $s=1$ на многообразии $S$ потоки коммутируют.

Аналогично нетрудно проверить, что условие $v_{x}+\left.i w\right|_{y=0}=0$ совместимо с симметрией (27) только при выполнении равенства $u(0)=0$.

Найдем условие обрыва, двойственное краевому условию $\left.w\right|_{y=0}=0$. Дифференцируя последнее, в силу уравнений (27) находим $w_{t}=-2 i\left(s_{x x} r-s r_{x x}\right)=0$. $\mathrm{B}$ результате несложных выкладок отсюда получаем $\left.(r / s)\right|_{y=0}=$ const, что с точностью до сдвигов переменной $u$ совпадает с равенством $u(0)=-u(-1)$. 


\section{§4. Преобразование Беклунда и граничные условия}

Напомним, что уравнение КП допускает одевающее преобразование, определяемое как линейное преобразование, действующее в классе решений уравнения (10) по правилу

$$
\phi_{1}=\left(D_{x}+\beta\right) \phi,
$$

где $\beta=\frac{1}{2} D_{x}^{-1}\left(v_{1}-v\right)$ и

$$
\phi_{1 x x}=i \phi_{1 y}+v_{1} \phi_{1} .
$$

При этом соответствующее преобразование Беклунда имеет вид нелокального уравнения в частных производных

$$
v_{x}+v_{1 x}=\left(v-v_{1}\right) D_{x}^{-1}\left(v-v_{1}\right)+i \theta D_{x}^{-1}\left(v_{y}-v_{1 y}\right),
$$

где $v=v(x, y, \tau)$ и $v_{1}=v_{1}(x, y, \tau)$ - решения уравнения КП, $\theta=1$. Преобразование Беклунда вида (33) с $\theta=-1$ определяется уравнением вида (31), в котором вместо $\phi_{1}$ и $\phi$ следует взять решения $\psi_{1}$ и $\psi$ сопряженных уравнений $\psi_{1 x x}=-i \psi_{1 y}+v_{1} \psi_{1}$ и $\psi_{x x}=-i \psi_{y}+v \psi$. Одевающее преобразование позволяет по известному решению $v$ уравнения КП строить его новое решение. Действительно, пусть $\phi-$ некоторое решение уравнения (10). Выберем $\beta=-D_{x} \ln \phi$ и воспользуемся формулой $v_{1}=v+2 D_{x} \beta=v-2 D_{x}^{2} \ln \phi$.

Предположим теперь, что решения $v$ и $v_{1}$ уравнения КП, связанные преобразованием Беклунда (33), удовлетворяют следующему дополнительному условию инволюции: $v_{1}(x, y, \tau)=v(x,-y, \tau)$. Существование таких решений можно доказать, пользуясь тем, что как само уравнение КП, так и его преобразование Беклунда не меняются при замене знака $y$. Дифференцируя приведенное выше условие инволюции, приходим к равенствам $v_{1 x}(x, y, \tau)=v_{x}(x,-y, \tau)$ и $v_{1 y}(x, y, \tau)=-v_{y}(x,-y, \tau)$. В силу найденных соотношений из формулы (33) при $y=0$ вытекает равенство $v_{x}(x, 0, \tau)=-i \theta D_{x}^{-1} v_{y}(x, 0, \tau)$, совпадающее $\mathrm{c}$ краевыми условиями (14.2), (14.4), так как $\theta= \pm 1$.

\section{§5. Модифицированное уравнение КП}

Найдем краевые условия для модифицированного уравнения КадомцеваПетвиашвили (мКП), впервые появившегося в работах $[16,17]$

$$
\begin{gathered}
v_{t}+v_{x x x}-\frac{3}{2} v^{2} v_{x}+3 \sigma^{2} w_{y}-3 \sigma v_{x} w=0, \\
w_{x}=v_{y},
\end{gathered}
$$

где $\sigma^{2}= \pm 1$. Уравнение (34) является условием существования совместного решения следующей пары линейных уравнений

$$
\begin{gathered}
\sigma \phi_{y}+\phi_{x x}+v \phi_{x}=0 \\
\phi_{\tau}+4 \phi_{x x x}+6 v \phi_{x x}+\left(3 v_{x}-3 \sigma w+\frac{3}{2} v^{2}\right) \phi_{x}+\delta \phi=0,
\end{gathered}
$$

где $\delta$ - произвольная постоянная.

Согласно тесту, предложенному в $\S 1$, требуется найти точечные дискретные симметрии уравнения (34). Легко видеть, что уравнение (34) не изменяется при 
замене $y \rightarrow-y, v \rightarrow-v$. При этой замене пара Лакса (35), (36) приводится к виду

$$
\begin{gathered}
\sigma \psi_{y}-\psi_{x x}+v \psi_{x}=0, \\
\psi_{\tau}+4 \psi_{x x x}-6 v \psi_{x x}-\left(3 v_{x}+3 \sigma w-\frac{3}{2} v^{2}\right) \psi_{x}+\delta \psi=0,
\end{gathered}
$$

ПрЕДЛОЖЕНИЕ 5. Пусть существует дифференциалъный оператор вида $M=\alpha D_{x}+\beta$, такой, что при $y=0$ для любого решения $\psi$ уравнения (38) функиия $\phi=M \psi$ является решением уравнения (36). Тогда переменные $v$ u связань одним из следующих соотношений:

$$
\begin{aligned}
& \left.v\right|_{y=0}=0, \\
& \left.\left(\sigma w_{y}+v v_{y}+v_{x y}\right)\right|_{y=0}=0 .
\end{aligned}
$$

При этом соответствующий оператор $M$ имеет вид

$$
\begin{aligned}
& M=1, \\
& M=\alpha D_{x}, \quad \text { əде } \alpha_{x}=-v \alpha .
\end{aligned}
$$

Если в предложении 5 поменять местами функции $\phi$ и $\psi$, то получим еще одно краевое условие

$$
\left.\left(\sigma w_{y}+v v_{y}-v_{x y}\right)\right|_{y=0}=0 .
$$

При этом соотношение $\psi=\alpha D_{x} \phi$, задающее соответствующий оператор, будет выполняться, только если $\alpha_{x}=v \alpha$.

ДокАЗАТЕЛЬСТво ПРЕДЛОжЕНИЯ 5. Так же как и при доказательстве предложения 2, введем дифференциальные операторы $A_{1}=-4 D_{x}^{3}-6 v D_{x}^{2}-b D_{x}-\delta$ и $A_{2}=-4 D_{x}^{3}+6 v D_{x}^{2}-k D_{x}-\delta$, где $b=3 v_{x}-3 \sigma w+\frac{3}{2} v^{2}, k=-3 v_{x}-3 \sigma w+\frac{3}{2} v^{2}$. Тогда уравнения (36) и (38) запишутся в виде $\phi_{\tau}=A_{1} \phi$ и $\psi_{\tau}=A_{2} \psi$. Искомый оператор удовлетворяет соотношению $M_{\tau}=A_{1} M-M A_{2}$, равносильному следующей переопределенной системе дифференциальных уравнений:

$$
\begin{gathered}
\alpha_{x}+\alpha v=0, \\
\alpha_{x x}+\alpha_{x} v+\alpha v_{x}+\beta_{x}+\beta v=0, \\
\alpha_{x x x}+\frac{3}{2} v \alpha_{x x}+\frac{1}{4} b \alpha_{x}+3 \beta_{x x}+3 v \beta_{x}+\frac{3}{2} \beta v_{x}-\frac{1}{4} k_{x} \alpha=-\frac{1}{4} \alpha_{t}, \\
\beta_{x x x}+\frac{3}{2} v \beta_{x x}+\frac{1}{4} b \beta_{x}=-\frac{1}{4} \beta_{t} .
\end{gathered}
$$

Из первых двух уравнений следует, что $\beta_{x}+\beta v=0$. Поэтому можно исключить из двух последующих уравнений старшие производные функций $\alpha$ и $\beta$ и переписать их в виде

$$
\begin{aligned}
& \beta_{t}=W \beta, \\
& \alpha_{t}=\left(W-k_{x}\right) \alpha+6 v_{x} \beta,
\end{aligned}
$$

где $W=4 v_{x x}-6 v v_{x}-2 v^{3}+v b$. Условие совместности уравнения (42) и уравнения $\beta_{x}+\beta v=0$ имеет вид $\left(W_{x}+v_{t}\right) \beta=0$. Пусть сначала равен нулю первый сомножитель, т. е. $v_{t}=-W_{x}$. Сравнивая его с уравнением мКП, приходим к равенству $v=0$. На функцию $\alpha$ получаем пару уравнений $\alpha_{x}=0$ и $\alpha_{t}=-3 \sigma v_{y} \alpha$, откуда $\alpha=0$, так как $v_{x y}=0$. Приравняем к нулю второй сомножитель, т. е. пусть $\beta=0$. При этом условие совместности уравнения (43) и уравнения $\alpha_{x}+\alpha v=0$ имеет вид $\left(W_{x}-k_{x x}+v_{t}\right) \alpha=0$. Случай $\alpha=0$ приводит к тривиальному ответу 
$M=0$. Поэтому в этом произведении приравняем нулю первый сомножитель: $W_{x}-k_{x x}+v_{t}=0$. Это уравнение можно переписать в виде

$$
\left.\left(-v_{t}-v_{x x x}+\frac{3}{2} v^{2} v_{x}+3 \sigma v v_{y}+3 \sigma v_{x} w+3 \sigma v_{x y}\right)\right|_{y=0}=0 .
$$

Сравнение уравнения (44) с исходным уравнением (34) показывает, что должно выполняться условие (39.2).

\section{§6. Уравнение Веселова-Новикова}

Для иллюстрации теста рассмотрим еще один пример, уравнение ВеселоваНовикова $(\mathrm{BH})$, возникшее как $1+2$-мерное обобщение уравнения Кортевега-де Фриза [18]. Оно имеет вид

$$
u_{t}=u_{x x x}+(c u)_{x}, \quad c_{y}=-3 u_{x},
$$

и является условием совместности следующей системы линейных дифференциальных уравнений (см., например, [14]):

$$
\begin{gathered}
\phi_{y}=\psi, \quad \psi_{x}=u \phi \\
\phi_{t}=\phi_{x x x}+c \phi_{x}, \quad \psi_{t}=u \phi_{x x}-u_{x} \phi_{x}+\left(u_{x x}+c u\right) \phi .
\end{gathered}
$$

Инволюция вида $y \rightarrow-y, u \rightarrow-u$ не меняет уравнения ВН, но меняет пару Лакса

$$
\begin{gathered}
\bar{\phi}_{y}=-\bar{\psi}, \quad \bar{\psi}_{x}=-u \bar{\phi} \\
\bar{\phi}_{t}=\bar{\phi}_{x x x}+c \bar{\phi}_{x}, \quad \bar{\psi}_{t}=-u \bar{\phi}_{x x}+u_{x} \bar{\phi}_{x}-\left(u_{x x}+c u\right) \bar{\phi}
\end{gathered}
$$

Пусть при $y=0$ уравнения (47) и (49) связаны линейным преобразованием вида

$$
\left(\begin{array}{l}
\phi \\
\psi
\end{array}\right)=M\left(\begin{array}{c}
\bar{\phi} \\
\bar{\psi}
\end{array}\right)
$$

где $M=\alpha D_{x}+\beta$ - дифференциальный оператор первого порядка, а коэффициенты $\alpha$ и $\beta$ - матричные функции от $x, t$. Тогда должно выполняться условие

$$
M_{t}=V M-M \bar{V}
$$

где

$$
V=\left(\begin{array}{cc}
D_{x}^{3}+c D_{x} & 0 \\
u D_{x}^{2}-u_{x} D_{x}+u_{x x}+c u & 0
\end{array}\right), \quad \bar{V}=\left(\begin{array}{cc}
D_{x}^{3}+c D_{x} & 0 \\
-u D_{x}^{2}+u_{x} D_{x}-u_{x x}-c u & 0
\end{array}\right) .
$$

Несложный анализ уравнения (51) показывает, что, во-первых, оно имеет двупараметрическое семейство решений вида

$$
M=\left(\begin{array}{cc}
0 & 0 \\
0 & \alpha_{22}
\end{array}\right) D_{x}+\left(\begin{array}{cc}
\beta_{11} & 0 \\
\alpha_{22} u & -\beta_{11}
\end{array}\right)
$$

без каких-либо ограничений на коэффициенты операторов $V$ и $\bar{V}$. А во-вторых, для существования трехпараметрического класса решений

$$
M=\left(\begin{array}{cc}
0 & 0 \\
0 & \alpha_{22}
\end{array}\right) D_{x}+\left(\begin{array}{cc}
\beta_{11} & 0 \\
0 & \beta_{22}
\end{array}\right)
$$


необходимо и достаточно выполнение условия связи $u=0$. Здесь $\alpha_{22}, \beta_{11}, \beta_{22}$ - произвольные постоянные. Следовательно, краевое условие $\left.u\right|_{y=0}=0$ согласуется с L-A-парой уравнения BH.

\section{§7. Заключение}

В статье не исследуется вопрос о том, как погрузить полученные краевые задачи в схему метода обратной задачи рассеяния (МОЗР). Ниже мы приводим некоторые соображения о том, почему проблема адаптации МОЗР к упомянутым краевым задачам в принципе разрешима.

Bo-nервыx, в случае двумеризованной цепочки Тоды все известные интегрируемые условия обрыва и только они проходят операторный тест, а они заведомо совместимы с МОЗР.

Bo-вторых, краевые условия для уравнения КП, найденные выше, проходят еще и хорошо апробированный симметрийный тест (см. предложение 3 выше, а также §3), являющийся уже менее формальным. Совместимость краевого условия с высшей симметрией фактически означает, что соответствующая краевая задача допускает явные частные решения, являющиеся одновременно стационарными решениями этой симметрии. И действительно, среди солитоноподобных решений уравнения КП2 выделяется широкий класс решений, подчиненных условиям $\left.\left(v_{x} \pm w\right)\right|_{y=0}=0$.

$B$-mpeтъих, операторный тест является непосредственным обобщением результатов работ $[4,5]$ по $1+1$-мерным уравнениям. В работе [5] на уровне примеров уравнений КдФ, sin-Gordon и уравнения Гарри Дима было обнаружено, что при наложении на уравнение интегрируемого краевого условия второе уравнение пары Лакса приобретает дополнительную дискретную симметрию, что было положено в основу теста на интегрируемость. Изложим кратко суть этого теста на нескольких примерах. Начнем с уравнения $\mathrm{K}_{\text {д }} u_{t}=u_{x x x}-6 u u_{x}$, которое совместимо с краевыми условиями $\left.u\right|_{x=0}=a,\left.u_{x x}\right|_{x=0}=b$, где $a$ и $b$ - произвольные действительные числа. Уравнение КдФ является условием совместности двух семейств линейных дифференциальных уравнений, «пронумерованных» спектральным параметром $\lambda \in \mathbb{C}$,

$$
Y_{x}=\left(\begin{array}{cc}
0 & 1 \\
u-\lambda & 0
\end{array}\right) Y, \quad Y_{t}=\left(\begin{array}{cc}
u_{x} & -4 \lambda-2 u \\
u_{x x}-(4 \lambda+2 u)(u-\lambda) & -u_{x}
\end{array}\right) Y .
$$

Вдоль прямой $x=0$ вторая из систем принимает вид

$$
Y_{t}(\lambda, t)=\left(\begin{array}{cc}
u_{x} & -4 \lambda-2 a \\
b-(4 \lambda+2 a)(a-\lambda) & -u_{x}
\end{array}\right) Y(\lambda, t) .
$$

Оно допускает инволюцию $\lambda \rightarrow h(\lambda), Y(\lambda, t) \rightarrow F(\lambda, t) Y(h(\lambda), t)$. Другими словами, функция $Y_{1}(\lambda, t)=F(\lambda, t) Y(h(\lambda), t)$ также является решением системы уравнений (52), если (см. [5]) $h(\lambda)=\left(-\lambda+\sqrt{c-3 \lambda^{2}}\right) / 2, c=3 a^{2}-b$,

$$
F(\lambda, t) \equiv F(\lambda)=\left(\begin{array}{cc}
2 \lambda+a & 0 \\
0 & 2 h+a
\end{array}\right) .
$$

Появление дополнительной инволюции позволяет линеаризовать уравнение эволюции матрицы рассеяния (см. [19]) и, тем самым, «проинтегрировать» соответствующую начально-краевую задачу. 
Для уравнения Гарри Дима $u_{t}+u^{3} u_{x x x}=0$ с парой Лакса

$$
\begin{aligned}
Y_{x}(x, t, \lambda) & =\left(\begin{array}{cc}
0 & 1 \\
-\lambda / u^{2} & 0
\end{array}\right) Y(x, t, \lambda) \\
Y_{t}(x, t, \lambda) & =-2 \lambda\left(\begin{array}{cc}
u_{x} & -2 u \\
u_{x x}+2 \lambda / u & -u_{x}
\end{array}\right) Y(x, t, \lambda)
\end{aligned}
$$

интегрируемым краевым условиям $u(0, t)=0, u_{x}(0, t)=b, b=$ const, соответствует инволюция $\lambda \rightarrow h(\lambda), Y(0, t, \lambda) \rightarrow F(\lambda, t) Y(0, t, h(\lambda))$ с функцией $h(\lambda)=\lambda$ и матрицей сопряжения

$$
F=I+\alpha e^{4 \lambda b t}\left(\begin{array}{ll}
0 & 0 \\
1 & 0
\end{array}\right), \quad \alpha=\text { const } .
$$

Другому типу интегрируемого краевого условия

$$
u_{x}(0, t)=a u, \quad u_{x x}(0, t)=\frac{a^{2}}{2} u+\frac{d}{u}, \quad a, d \in \mathbb{R},
$$

соответствует указанная выше инволюция с параметрами, заданными в виде

$$
F=\left(\begin{array}{cc}
\lambda & 0 \\
\frac{1}{2} a(\lambda-h(\lambda)) & h(\lambda)
\end{array}\right), \quad h=\frac{-d-2 \lambda \pm \sqrt{d^{2}-4 \lambda d-12 \lambda^{2}}}{4} .
$$

В случае нулевых (заведомо интегрируемых) краевых условий $u(0, t)=0$, $v(0, t)=0$ для системы

$$
i u_{t}=-u_{x x}+2 v u^{2}, \quad-i v_{t}=-v_{x x}+2 u v^{2},
$$

сводящейся к нелинейному уравнению Шрёдингера, второе уравнение пары Лакса

$$
\begin{aligned}
Y_{x}(x, t, \lambda) & =\left(\begin{array}{cc}
i \lambda & u \\
v & -i \lambda
\end{array}\right) Y(x, t, \lambda) \\
Y_{t}(x, t, \lambda) & =\left(\begin{array}{cc}
-2 i \lambda^{2}-i u v & -2 \lambda u+i u_{x} \\
-2 \lambda v-i v_{x} & 2 i \lambda^{2}+i u v
\end{array}\right) Y(x, t, \lambda),
\end{aligned}
$$

принимает вид

$$
Y_{t}(x, t, \lambda)=\left(\begin{array}{cc}
-2 i \lambda^{2} & i u_{x} \\
-i v_{x} & 2 i \lambda^{2}
\end{array}\right) Y(x, t, \lambda)
$$

и, очевидно, допускает инволюцию $\lambda \rightarrow-\lambda$.

Для $2+1$-мерных уравнений пара Лакса не содержит спектрального параметра. Точнее, можно считать, что спектральный параметр принимает конечное число значений. Действительно, все модели, рассмотренные выше, имели ровно две неэквивалентные (не связанные калибровочным преобразованием) пары Лакса. Поэтому в $2+1$-мерном случае инволюция $\lambda \rightarrow h(\lambda)$ есть не что иное, как переход к неэквивалентной L-A-паре. Матрица сопряжения $F(\lambda)$ заменяется дифференциальным оператором $M=M\left(D_{x}\right)$.

В примерах мы ограничились сопрягающими операторами $M=M\left(D_{x}\right)$ не выше второго порядка. С ростом порядка оператора вычислительные трудности быстро возрастают, а в краевых условиях появляются нелокальные переменные.

Авторы благодарят Р. И. Ямилова за полезные обсуждения. 


\section{ЛитеРАтУрА}

1. Склянин E. K. Граничные условия для интегрируемых систем. Функц. анализ и его прил., 21, вып. 2, 86-87 (1987).

2. Gürel B., Gürses M., Habibullin I. T. Boundary value problems, compatible with symmetries. Phys. Lett. A, 190, 231-237 (1994).

3. Бикбаев Р. Ф., Тарасов В. О. Неоднородная краевая задача на полуоси и на отрезке для уравнения sine-Gordon. Алгебра и анализ, 3, вып. 4 78-92 (1991).

4. Habibullin I. T., Kazakova T. G. Boundary conditions for integrable chains. J. Phys. A: Math. and Gen. 34, 10369-10376 (2001).

5. Habibullin I. T., Vil' danov A. N. Boundary conditions, consistent with L-A-pairs. In: Proc. of the International Conference Modern Group Analysis 2000 (V. A. Baikov, ed.), Ufa, 2001, pp. 80-81.

6. Хабибуллин И. Т. Граничные задачи на полуплоскости для уравнения Ишимори, совместимые с методом обратной задачи рассеяния. ТМФ, 91, №3, 363-376 (1992).

7. Лезнов A. Н., Савелъев M. В. Групповые методы интегрирования нелинейных динамических систем. Наука, М., 1985.

8. Дегасперис А., Манаков С. В., Сантини П. М. Смешанные задачи для линейных и солитонных уравнений в частных производных. ТМФ, 133, №2, 184-201 (2002).

9. Кадомиев Б. Б., Петвиашвили В. И. Об устойчивости уединенных волн в слабо диспергирующих средах. ДАН СССР, 192, №4, 753-756 (1970).

10. Захаров В. Е., Манаков С. В., Новиков С. П., Питаевский Л. П. Теория солитонов. Метод обратной задачи. Наука, М., 1980.

11. Дрюма B. С. Об аналитическом решении двумерного уравнения Кортевега-де Вриза (КДВ). Письма в ЖЭТФ, 19, №12, 753-755 (1974).

12. Захаров B. E., Шабат A. Б. Схема интегрирования нелинейных уравнений математической физики методом обратной задачи теории рассеяния. Функц. анализ и его прил., 8, вып. 3, 43-53 (1974).

13. Гриневич П. Г. Преобразование рассеяния для двумерного оператора Шрёдингера при одной энергии и связанные с ним интегрируемые уравнения математической физики. Дисс. д.ф.-м.н., ИТФ, Черноголовка, 1999.

14. Адлер В. Э., Шабат А. Б., Ямилов Р. И. Симметрийный подход к проблеме интегрируемости. ТМФ, 125, №3, 355-424 (2000).

15. Shabat A. B. Higher symmetries of two-dimensional lattices. Phys. Lett. A, 200, No. 121 (1995).

16. Konopelchenko B. G. On the gauge-invariant description of the evolution equations integrable by Gelfand-Dikij spectral problems. Phys. Lett. A, 92, No. 323 (1982).

17. Jimbo M., Miwa T. Solitons and infinite dimensional Lie algebras. Publ. Res. Inst. Math. Sci., 19(3), No. 943 (1983).

18. Веселов А. П., Новиков С. П. Операторы Шрёдингера с конечнозонными двумерными потенциалами. Явные формулы и эволюционные уравнения. ДАН СССР, 279, 20 (1984).

19. Хабибуллин И. Т. Начально-краевая задача для уравнения КдФ на полуоси с однородными краевыми условиями. ТМФ, 130, №1. 31-53 (2002).

Институт математики Уфимского

Поступило в редакцию научного центра РАН

email: ihabib@imat.rb.ru 11 ноября 2002 г.

Уфимский государственный нефтяной

технический университет 